\title{
Three-Phase Characterization of Uniaxially Stretched Linear Low-Density Polyethylene
}

\author{
Blerina Kolgjini, Gustaaf Schoukens, and Paul Kiekens \\ Department of Textiles, Ghent University, Technologiepark 907, 9052 Zwijnaarde, Belgium \\ Correspondence should be addressed to Gustaaf Schoukens, gustaaf.schoukens@ugent.be
}

Received 31 March 2011; Revised 12 July 2011; Accepted 20 August 2011

Academic Editor: Haojun Liang

Copyright ( $) 2011$ Blerina Kolgjini et al. This is an open access article distributed under the Creative Commons Attribution License, which permits unrestricted use, distribution, and reproduction in any medium, provided the original work is properly cited.

\begin{abstract}
This study comprises a detailed morphological study of cold-drawn polyethylene monofilaments by Raman spectroscopy, differential scanning calorimetry (DSC) and X-ray measurements. The structure of the three-phase morphology of the linear lowdensity polyethylene monofilaments was investigated by combining these measurements. It was found that the most important structure variation was found in the intermediate or rigid amorphous phase, whereby the amounts of crystalline and amorphous phases were nearly constant and almost independent of the cold-draw ratio. The intermediate third phase contains gauche and transmolecules, and the amount of transmolecules was increased with the cold-draw ratio and was directly related to this cold-draw ratio. It was found that the two peaks in the Raman spectra, respectively, at 1303 and $1295 \mathrm{~cm}^{-1}$, can be correlated to the amount of gauche and transmolecules in the polyethylene monofilaments. A good and new insight into the three-phase morphology was obtained by combining the DSC and X-ray measurements with the amounts of trans- and gauche molecules from the Raman spectra analysis.
\end{abstract}

\section{Introduction}

Polyethylene has been one of the most extensively studied polymers, and the understanding of the structure-properties relationship has been one of the main topics of fundamental research over the past few decades. Linear low-density polyethylene (LLDPE) is used extensively in environmental applications, such as packaging and monofilaments for artificial turf applications.

Polyethylene in the solid state, as part of polyolefin family, is a semicrystalline polymer which consists of a three-phase morphology: a crystalline phase surrounded by a noncrystalline phase comprising a partially ordered layer (third phase) adjacent to the crystallites and a disordered phase (amorphous phase) in the intervening spaces [1-3]. The third phase or transition phase is an intermediate component in addition to the crystalline and amorphous phases. The character of the third phase, also referred to as the interface, the intermediate phase, or rigid amorphous phase, has been a subject of discussions in several papers. Raman spectroscopy was introduced to characterize the three-phase morphological structure of semicrystalline polyethylene [4]. Such investigations demonstrated that chains involved in the third phase or in the anisotropic disordered phase were stretched, but lacked lateral order. The same conclusion was also observed from other results [5], showing that the noncrystalline interlamellar phase is anisotropic and exhibits properties that are intermediate between that of a crystalline solid and of an amorphous melt.

The nonlinear stress strain behavior of polyethylene material is governed by the relative proportion of the crystalline and noncrystalline phases, consisting of the amorphous and oriented amorphous phase, their orientation, and their connectivity with respect to one another. Based on a nonlinear viscoelastic model [6], the amorphous phase is in a liquid-crystalline state. Young's modulus and the strength of semicrystalline polymers are primarily affected by quasiamorphous interlamellar regions [7] and contained/consisted of several types of molecules, such as loops, tails, and bridges which joined-up to lamellae can be distinguished. The intermediate phase, which may be summarized as being similar to linking lamellae and 
amorphous [8], forms a surface layer around each lamellae acting as a wrapping membrane. Some authors [9] assumed the intermediate phase has mechanical properties slightly stiffer than a purely amorphous phase. The larger the lengthto-thickness ratio of the lamellae, the stronger the reinforcing effects of the lamellae on the amorphous matrix [10].

During uniaxial deformation, polymeric materials are often subjected to large plastic deformations, giving rise to preferential orientation of macromolecules and morphology, which may result in a high anisotropy of the structure, thus resulting in mechanical properties. Furthermore, especially in thin films, a preferred orientation of the crystalline component produced by transcrystallization during cooling and/or by spin casting may give rise to strong anisotropy and, therefore, can have a profound influence on the mechanical properties of these films [11-13]. Normally, uniaxially oriented filaments $[4,9]$ show good mechanical properties in the orientation direction and their relaxation behavior is explained by the presence of amorphous phase. If the third phase is described as a rigid amorphous phase and by accepting the presence of these phase, it was possible to compute the effective elastic properties of polymeric materials.

The Raman spectra can be used to assess the level of the three morphological components, by giving cumulative information with respect to all phases [4, 14-18]. According to Glotin and Mandelkern [14], Raman spectra are one of the few available techniques which allow for an assessment of the interfacial concentration and its structure. As it is described [15], the internal normal modes between 1000 and $16000 \mathrm{~cm}^{-1}$ are frequently used to study morphological structure and can be divided into three vibration areas: $\mathrm{C}-\mathrm{C}$ stretching between 1000 and $1200 \mathrm{~cm}^{-1}$ — sensitive to molecular orientation, stress and conformation; the $-\mathrm{CH}_{2}-$ twisting vibrations around $1295 \mathrm{~cm}^{-1}$ — can be used as an internal standard; and the $-\mathrm{CH}_{2}$ - bending modes between 1400 and $1470 \mathrm{~cm}^{-1}$ —sensitive to chain packing (the $1415 \mathrm{~cm}^{-1}$ bands is assigned to orthorhombic crystallinity).

X-ray measurements provide a model of three-phase morphology [19]. Wide angle diffraction (WAXS) scans the sample, and the scattering intensity is plotted as function of the angular position $2 \theta$. The results of the X-ray measurements suggested that the amorphous halo of a polyethylene in a solid state is the sum of scattering from a completely amorphous, liquid-like phase and from the intermediate, better-ordered regions that originate during crystallization.

The structure of the interphase, described as anisotropic [5] and having properties intermediate between that of semicrystalline solid and amorphous melt, is still not clear; however, The objective of this work is to obtain a better insight into the structure of the third phase by combining DSC, Raman, and X-ray measurements. The structure of the intermediate or rigid amorphous is changed by uniaxially stretching the polyethylene samples. For this reason, several monofilaments of linear low density polyethylene with different draw ratios were investigated and the results analyzed.

\section{Materials and Methods}

2.1. Materials and Preparation. In this study, the polymer chosen is linear low-density polyethylene (LLDPE) provided by DOWLEX $2035 \mathrm{G}$ with a density of $0.919 \mathrm{~g} / \mathrm{cm}^{3}$, a melt index of $6 \mathrm{~g} / 10 \mathrm{~min}$. This LLDPE is an ethylene-octene copolymer with a molecular weight $\mathrm{M}_{w}$ of 66000 and $\mathrm{M}_{w} / \mathrm{M}_{n}$ of 3.22 .

The filaments were produced by extrusion on a Haake Polydrive Extruder (Thermo Electronic Corporation). It is a single screw extruder of $25 \times D$ in length and a screw diameter of $19 \mathrm{~mm}$, with a 3-zone heating system. The temperature in the die $(T)$ was $220^{\circ} \mathrm{C}$. The die has 5 diamond-shaped openings with a cross section of $2.36 \mathrm{~mm}^{2}$ each. The monofilaments were stretched in the molten stage (MDR) and pulled directly afterwards through a water bath. The obtained monofilaments were conditioned in air oven at $95^{\circ} \mathrm{C}$ and stretched to different draw ratios (CDR). The total draw ratio (TDR) is the same for all the products and is calculated by multiplying the melt draw ratio (MDR) with the cold-draw ratio (CDR). The melt and cold-draw ratios are summarized in Table 1 for all the produced samples. The final section of all the monofilaments was equal to $0.09 \mathrm{~mm}^{2}$.

2.2. Characterization Methods. Uniaxial tensile deformation was performed with a Instron 3369 tensile apparatus with a load cell $500 \mathrm{~N}$. The length of the starting sample between the Instron clamps was $50 \mathrm{~mm}$. A constant deformation rate, $500 \mathrm{~mm} / \mathrm{min}$, was applied to the sample throughout the tensile deformation. For each sample, 5 tensile deformations were done and the elastic modulus was calculated from the mean values of these 5 tensile deformations. The deformation experiments were carried out at room temperature. The stress and strain obtained during the tensile deformations were the engineering stress and engineering strain measured directly between the clamps.

Differential Scanning Calorimetry (DSC) was performed on a DSC Q 2000 (TA Instruments), with a standard heating rate of $10^{\circ} \mathrm{C} / \mathrm{min}$ in a nitrogen environment.

Calibration of temperature and melting enthalpy was performed with an indium and tin sample. An enthalpy of $290 \mathrm{~J} / \mathrm{g}$ for perfect crystalline polyethylene was used to calculate the percentage of crystallinity $\left(\mathrm{CRY}_{\mathrm{DSC}}\right)$ using the following:

$$
\% \mathrm{CRY}_{\text {DSC }}=\frac{\left(100 * \Delta H_{\mathrm{exp}}\right)}{\Delta H^{\circ}},
$$

where $\Delta H_{\exp }$ is the experimentally determined heat of fusion and $\Delta H^{\circ}$ is the heat of fusion the perfect crystalline polyethylene.

Raman measurements were performed on an FT-Perkin Elmer instrument. The measurements range is from 3500 to $300 \mathrm{~cm}^{-1}$. Three repetitions were performed for each sample, consisting of 32 scans, and a laser power of $800 \mathrm{~mW}$ was used. The raw Raman spectra were smoothed, and baseline corrected.

WAXS measurements where done on an ARL-XTRA, X-ray diffractometer from Thermo Fisher Scientific at 
TABLE 1: The melt draw ratio (MDR) and cold-draw ratio (CDR) of the obtained monofilaments. The total draw ratio (TDR) is calculated by multiplying MDR with CDR.

\begin{tabular}{lccc}
\hline Production & MDR & CDR & TDR \\
\hline A-3 & 3,5 & 7,2 & 25,2 \\
A-4 & 4,1 & 6,2 & 25,4 \\
A-5 & 4,5 & 5,7 & 25,7 \\
A-6 & 5,0 & 5,0 & 25,0 \\
A-7 & 5,7 & 4,5 & 25,7 \\
A-8 & 6,0 & 4,2 & 25,2 \\
A-9 & 6,8 & 3,7 & 25,2 \\
\hline
\end{tabular}

the COMOC research group (University of GENT). Such measurements are used to characterize the crystalline microscopic structure of the polymer. The radiation source $\mathrm{Cu}$ $\mathrm{K} 1$ was operated at $45 \mathrm{kV} 44 \mathrm{~mA}$. The scanning angle ranged from $5^{\circ}$ to $50^{\circ}(2 \theta), \lambda=1.54 \AA, 0.02$ step-size. The percentages of amorphous, orthorhombic crystalline phase, and the monoclinic phase were calculated after the decomposition of the original spectra using the Gaussian fit procedure.

\section{Results and Discussion}

3.1. Stress-Strain Curves. The tensile behavior of the LLDPE monofilaments with different draw ratios was studied at room temperature. Figure 1 shows the engineering stressstrain curves of the different LLDPE monofilaments recorded during uniaxial tensile deformation at room temperature. For each sample, the elastic modulus and maximum tensile force in the stretching experiments were calculated from the stress-strain curves represented on Figure 1 and the results are summarized in Table 2.

As can be seen from Table 2, the Elastic modulus calculated as a slope of stress strain curves is increasing by increasing the draw ratio. The orientation, induced by drawing, has a marked effect on the properties of crystalline polymers and cannot be explained only by the degree of crystallinity in the oriented samples, but seems to be more related to the structural changes in the intermediate phase.

3.2. DSC Measurements. From DSC measurements, we observed that the range of the melting temperatures and final melting temperature was nearly constant, independent of the draw ratio, as represented in Figure 2. The DSC melting endotherms are characterized by a broad melting range of temperatures between $30^{\circ} \mathrm{C}$ and $144^{\circ} \mathrm{C}$. Such a broad melting range of temperatures are characteristic of LLDPE materials and are the result of the presence of a broad distribution of crystal sizes. This is further attributed to a highly heterogeneous structure that results from nonrandom incorporation of the comonomer during the polymerization with a Ziegler-Natta catalyst. The total melting range, from $30^{\circ} \mathrm{C}$ up to $144^{\circ} \mathrm{C}$, was used to calculate the melting enthalpy and the corresponding calculated degree of crystallinity. The percentage of crystallinity is only increasing with

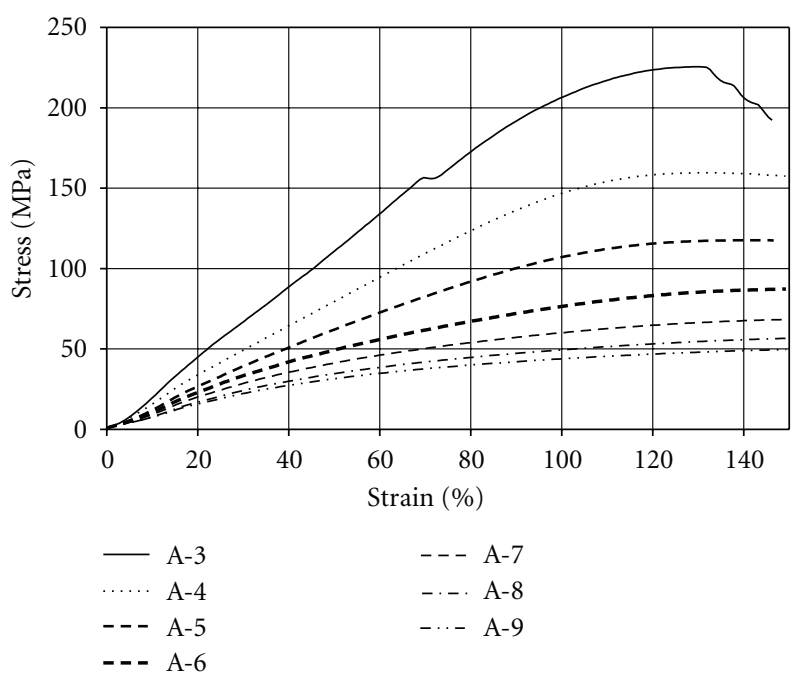

FIGURE 1: Engineering stress-strain curves of the LLDE monofilaments at room temperature.

TABle 2: Classical tensile properties (Elastic Modulus and Maximum Tensile force) and crystallinity by DSC of the oriented LLDPE monofilaments.

\begin{tabular}{lcccc}
\hline \multicolumn{2}{c}{ Cold-draw ratio } & $\begin{array}{c}\text { E Modulus } \\
(\mathrm{MPa})\end{array}$ & $\begin{array}{c}\text { Max Tensile } \\
\text { force (N) }\end{array}$ & $\begin{array}{c}\text { DSC } \\
\text { Crystallinity } \\
(\%)\end{array}$ \\
\hline A-3 & 7.2 & $270 \pm 3$ & $100 \pm 9$ & $51 \pm 1$ \\
A-4 & 6.1 & $203 \pm 8$ & $75 \pm 5$ & $50 \pm 2$ \\
A-5 & 5.7 & $165 \pm 9$ & $57 \pm 4$ & $47 \pm 0$ \\
A-6 & 5.0 & $144 \pm 6$ & $59 \pm 6$ & $47 \pm 2$ \\
A-7 & 4.5 & $125 \pm 4$ & $55 \pm 5$ & $47 \pm 1$ \\
A-8 & 4.2 & $112 \pm 4$ & $53 \pm 2$ & $46 \pm 3$ \\
A-9 & 3.7 & $103 \pm 3$ & $45 \pm 3$ & $46 \pm 0$ \\
\hline
\end{tabular}

a small fraction by increasing the draw ratio. The results for the crystallinity fraction $\left(\mathrm{CRY}_{\mathrm{DSC}}\right)$, the amorphous and intermediate phase calculated by using $\left(1-\mathrm{CRY}_{\mathrm{DSC}}\right) * 100 \%$, are summarized in Table 2. From the DSC curves (see Figure 2), it is quite clear that the highest melting peak temperature is around $123^{\circ} \mathrm{C}$ for all the samples; however, they show slightly different values for the melting enthalpy as a result of the cold drawing.

3.3. Raman Spectra. Figure 3 shows some of the recorded Raman spectra in the region of $950-1500 \mathrm{~cm}^{-1}$ and corresponding to the spectra commonly observed for semicrystalline polyethylenes.

As represented on Figure 4, the measured Raman spectra were decomposed into individual bands using Gauss functions in the region between 1250 and $1500 \mathrm{~cm}^{-1}$. A very good approximation of the measured spectra by these Gauss deconvolution, using the indicated individual bands, is obtained as indicated in Figure 4.

The total integral intensity $I_{\mathrm{TW}}$ of the $\mathrm{CH}_{2}$-twisting region $\left(1250-1350 \mathrm{~cm}^{-1}\right)$ is independent from the degree 


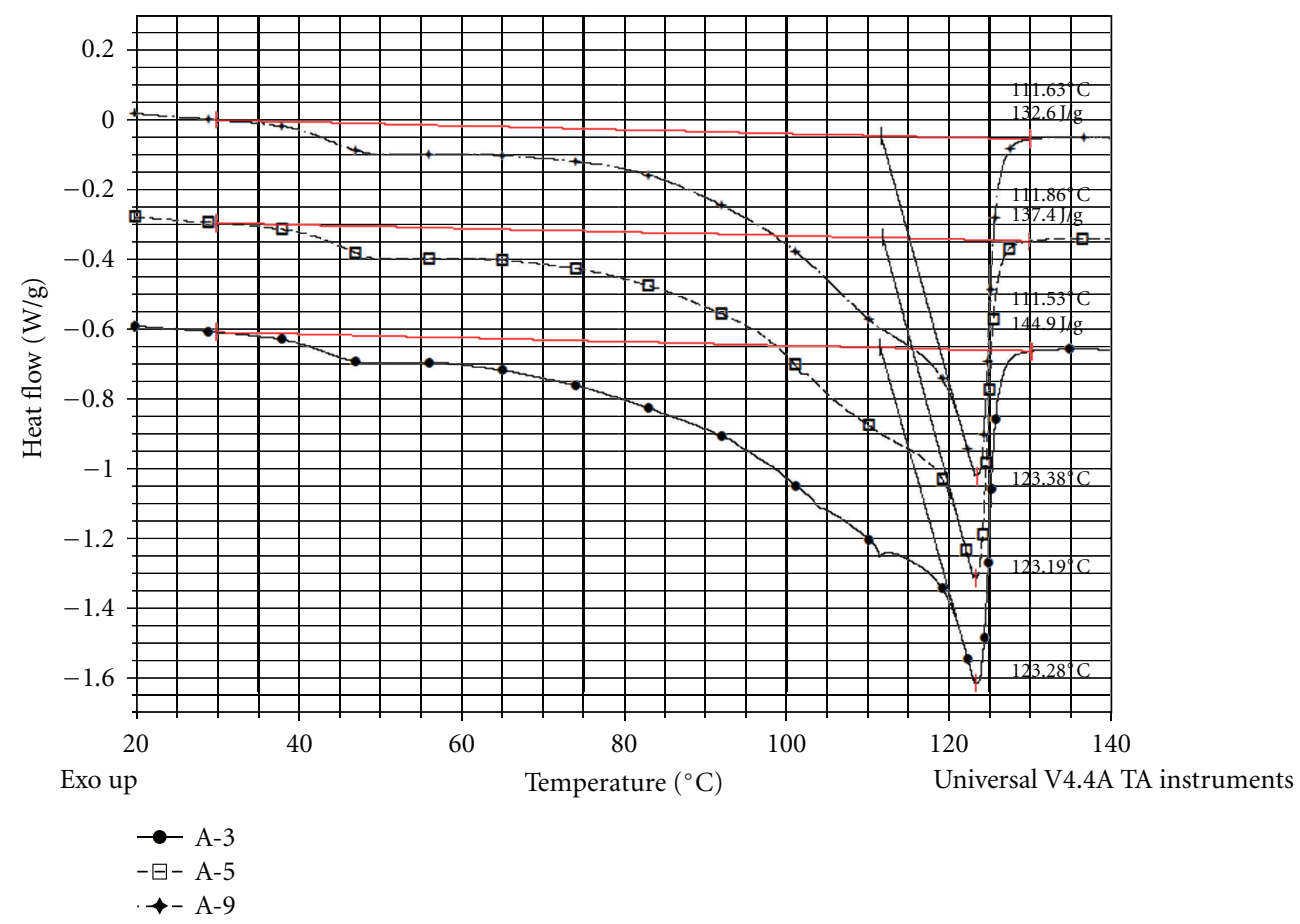

Figure 2: DSC curves for samples A-3, A-5, and A-9 at a melting stage.

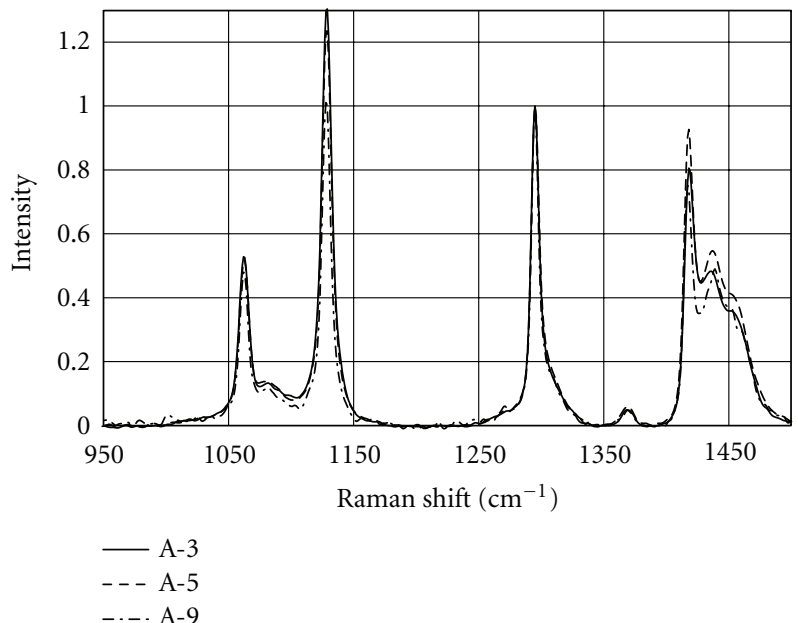

FIGURE 3: Raman spectra of some LLDPE monofilaments.

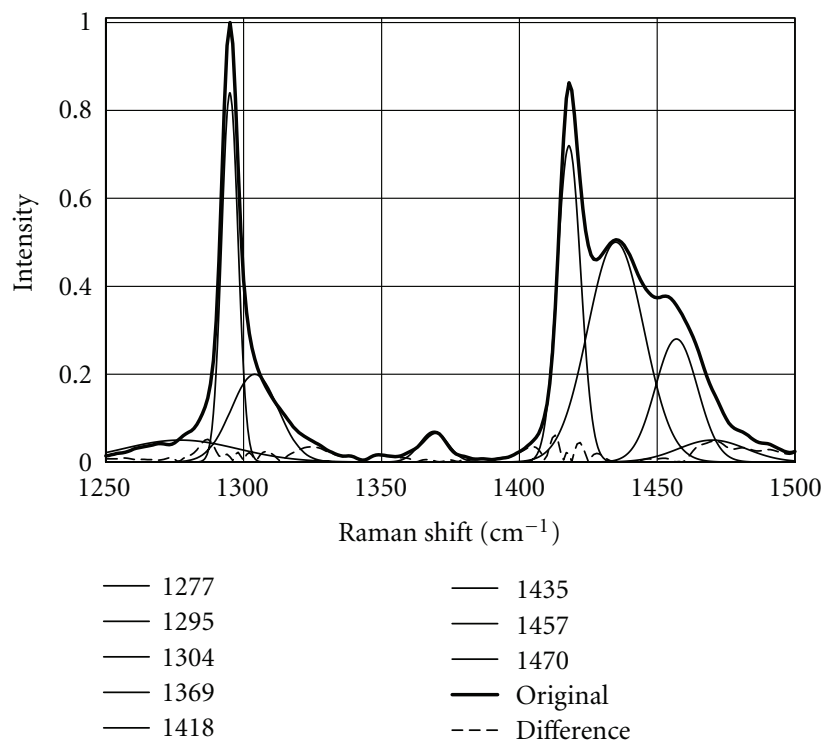

Figure 4: Gauss deconvolution of Raman spectra (Sample A-3).

of crystallinity and is used as an internal standard [16]. The spectrum in this twisting region can be deconvoluted into a narrow band centered at $1295 \mathrm{~cm}^{-1}$ and a broader component having its maximum intensity at $1303 \mathrm{~cm}^{-1}$. In the classical approach [16], the mass fraction of the crystalline phase $\left(C_{R}\right)$ is calculated using the integral intensity of the band located at $1416 \mathrm{~cm}^{-1}$, and the mass fraction of the amorphous phase $\left(A_{R}\right)$ is calculated using the integral intensity of the band located at $1303 \mathrm{~cm}^{-1}$. In another approach, the integral intensity of the band located at $1295 \mathrm{~cm}^{-1}$ is used to calculate all transmolecules [15].
The structures calculated from these Raman approaches are not necessarily identical with structures formed during crystallization and drawing of LLDPEs, as characterized by the other analyzing methods. Based on these published results with the cited approaches, we define the amount of the gauche-conformations as the ratio between the total integral intensity at $1303 \mathrm{~cm}^{-1}\left(I_{1303}\right)$, relative to $I_{\mathrm{TW}}$ and the corresponding amount of the transconformations as the 


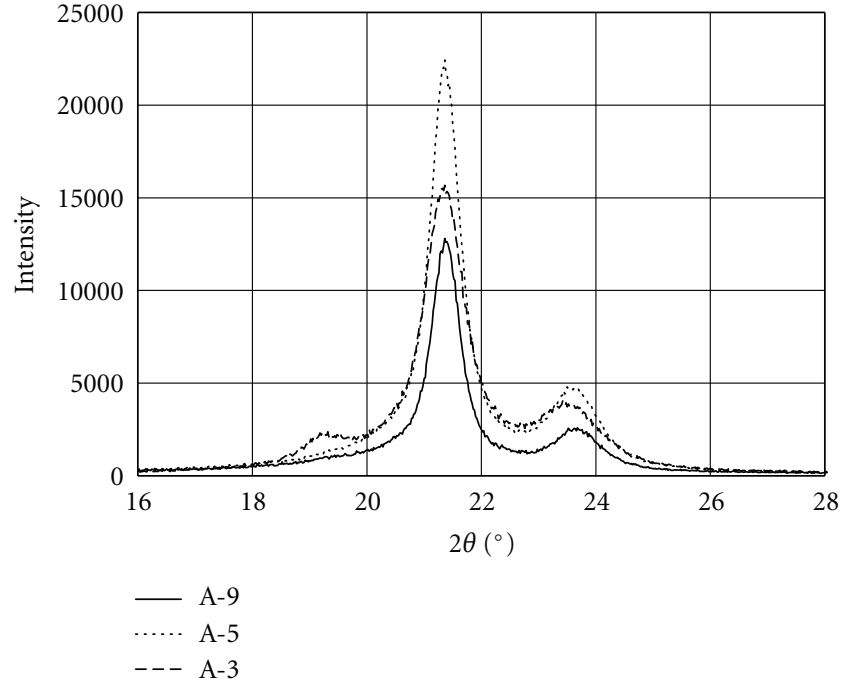

Figure 5: Measured X-ray spectra of some LLDPE monofilaments.

ratio between the total integral intensity at $1295 \mathrm{~cm}^{-1}\left(I_{1295}\right)$ relative to $I_{\mathrm{TW}}$, according to the following:

$$
\begin{gathered}
\text { \%Trans }=\left(\frac{I_{1295}}{I_{\mathrm{TW}}}\right) * 100, \\
\% \text { Gauche }=\left(\frac{I_{1303}}{I_{\mathrm{TW}}}\right) * 100 .
\end{gathered}
$$

The results calculated for the different LLDPE monofilaments are summarized in Table 3.

3.4. X-Ray Spectra. The recorded X-ray spectra were used to calculate the amount of the amorphous phase in the different LLDPE monofilaments after the Gauss decomposition of diffraction. The characteristic diffractions are presented in Figure 5 and the peak band of the amorphous phase at $19.5^{\circ}$, which is typical for polyethylene polymer, was used to calculate the amount of the amorphous phase. The results of the amount of amorphous phase are summarized in Table 3.

The result of decomposition for sample A3 is shown in Figure 6. Both crystalline peaks and amorphous halos were represented by Gauss profiles. These results showed that the monoclinic or triclinic phase was present in the cold-drawn material. However, the amount of monoclinic material, determined by X-ray measurements, is rather small. But the presence of this monoclinic material is an indication of the presence of a partially ordered component of the polymer structure, with a lower degree of order than the true crystalline structure.

The results obtained from DSC, Raman, and X-ray measurements are summarized in Table 3, together with the amount and structure of the 3rd phase calculated as the difference between the noncrystalline structures from DSC and the amorphous fraction from X-ray measurements.

3.5. Third-Phase Characterization. The Raman measurements together with the X-ray and DSC measurements

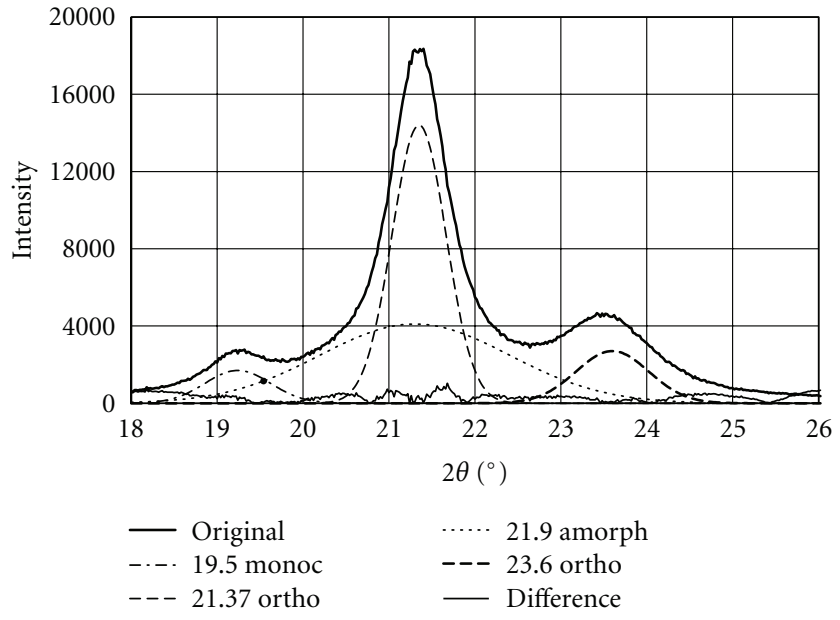

Figure 6: Gauss curve fitting for X-ray spectra (Sample A-3).

will be used to obtain a better and further insight in the amount and structure of the noncrystalline fraction of the monofilaments. The crystalline phase is obtained from the DSC measurements calculated by using formula (1) and the difference $\left(100 \%-\mathrm{CRY}_{(\mathrm{DCS})}\right)$ is the noncrystalline phase containing the amorphous and intermediate or 3rd phase. The content of gauche and transmolecules is obtained from the Raman measurements. The crystalline phase, calculated from the DSC measurements, contains only transconformations. The amorphous phase, containing only gauche molecules and described as a mobile gauche-containing amorphous component [20], was calculated from the Xray measurements. The difference between the noncrystalline fraction calculated from DSC and the amorphous phase calculated by X-ray yields the resulted percentage of intermediate phase presented in the different samples. The percentage of the 3rd phase is nearly constant, only decreasing a little bit by the highest draw ratios.

The differences between the gauche content from Raman and the fraction of the amorphous phase by X-ray were calculated, and these values correspond with the amount of gauche molecules present in the $3 \mathrm{rd}$ phase. The content of transmolecules increases with the draw ratio; and the maximum draw ratio is obtained if the 3rd phase contained only transmolecules. At this limit, all the polymer chains in the 3rd phase are completely stretched in the draw ratio.

The structure of the 3rd phase can be characterized by combining the DSC, Raman, and X-ray measurements. The basic assumptions hereby is that the Raman $\mathrm{CH}_{2}$-twisting vibration can be split up into two bonds, one corresponding with the trans- $\left(I_{1295}\right)$ molecules and the one with the gauche molecules $\left(I_{1303}\right)$. As a result, the splitting of the $\mathrm{CH}_{2}-$ twisting region in the Raman spectra corresponds to the splitting of the conformers into trans- and gauche molecules and not into amorphous and ordered structures. This represents a major difference with the published interpretations of the Raman spectra.

3.6. Mechanical Properties Related to the Morphology. Upon stretching, the amount of the transsegments in the $3 \mathrm{rd}$ 
TABLE 3: Structure analysis of the three phases of the LLDPE monofilaments.

\begin{tabular}{|c|c|c|c|c|c|c|c|c|}
\hline \multirow{2}{*}{ CDR } & \multicolumn{2}{|r|}{ DSC } & \multicolumn{2}{|c|}{ Raman } & \multicolumn{2}{|l|}{ X-ray } & \multicolumn{2}{|c|}{ 3rd phase } \\
\hline & $\begin{array}{c}\text { CRY } \\
(\mathrm{T})(\%)\end{array}$ & $\begin{array}{l}\text { Amorphous+ } \\
\text { 3rd phase (\%) }\end{array}$ & $\begin{array}{c}\text { Transstructure } \\
(\%)\end{array}$ & $\begin{array}{c}\text { Gauche } \\
\text { structure } \\
(\%)\end{array}$ & $\begin{array}{c}\text { Amorphous } \\
(\%)\end{array}$ & $\begin{array}{c}\text { Trans } \\
(\%)\end{array}$ & $\begin{array}{c}\text { Gauche } \\
(\%)\end{array}$ & $\begin{array}{c}\text { 3rd phase } \\
(\%)\end{array}$ \\
\hline 7.2 & $51 \pm 1$ & 49 & 64 & 36 & $36.0 \pm 2.2$ & 13.0 & 0.0 & 13.0 \\
\hline 6.1 & $50 \pm 2$ & 50 & 59 & 41 & $36.0 \pm 1.9$ & 9.0 & 5.0 & 14.0 \\
\hline 5.7 & $47 \pm 0$ & 53 & 54 & 46 & $38.9 \pm 3$ & 7.0 & 7.1 & 14.1 \\
\hline 5.0 & $47 \pm 2$ & 53 & 53 & 47 & $36.3 \pm 1.7$ & 6.0 & 10.7 & 16.7 \\
\hline 4.5 & $47 \pm 1$ & 53 & 51 & 49 & $37.1 \pm 2.1$ & 4.4 & 11.5 & 15.9 \\
\hline 4.2 & $46 \pm 3$ & 54 & 50 & 50 & $36.5 \pm 4$ & 4.0 & 13.5 & 17.5 \\
\hline 3.7 & $46 \pm 0$ & 54 & 49 & 51 & $36.6 \pm 4$ & 3.0 & 14.4 & 17.4 \\
\hline
\end{tabular}

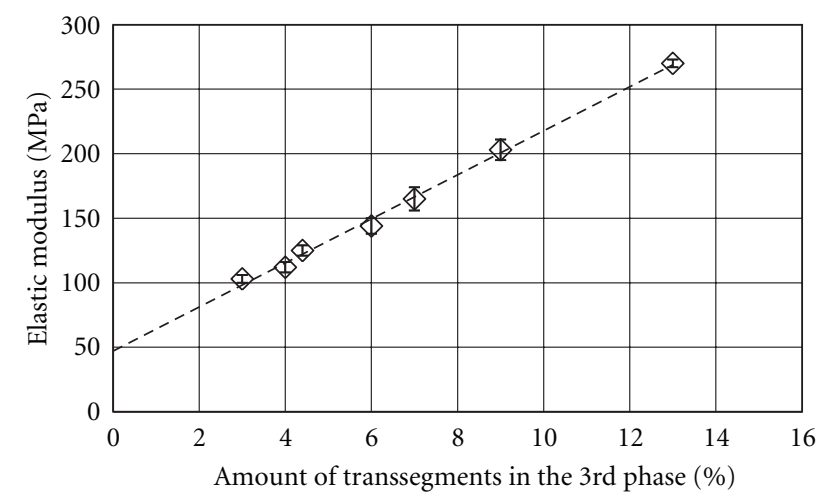

FIgURE 7: Elasticity modulus as a function of the amount of transsegments in the LLDPE monofilaments.

phase increases, by a similar amount of the crystalline and amorphous phase. This is related to the extension of the tiemolecules in the 3rd phase and has a direct influence on the mechanical properties of the oriented monofilaments. Figure 7 shows the influence of the amount of the trans- and gauche segments on the elasticity modulus of the oriented monofilaments.

The elasticity modulus is a linear function of the amount of transsegments in the intermediate phase, starting from a value of $45 \mathrm{MPa}$ for the intermediate phase containing $100 \%$ of gauche molecules and increasing to $280 \mathrm{MPa}$ for $100 \%$ transsegments. The 3rd phase is the interconnecting phase between the crystallites, and the mechanical properties of this 3 rd phase are directly related to the overall mechanical properties of the monofilaments. At/Using this limit, the monofilaments contain $13 \%$ of third phase and an elasticity modulus of $280 \mathrm{MPa}$, which give an elasticity modulus of the third phase of $2150 \mathrm{MPa}$.

\section{Conclusions}

LLDPE monofilaments with different cold-drawn ratios were produced, and the three-phase morphology was characterized. The three-phase morphology contains a crystalline phase, an amorphous phase, and a 3rd, or intermediate, phase. The combination of Raman spectroscopy, DSC, and
$\mathrm{X}$-ray measurements creates the possibility to characterize the amounts and composition of the three phases. The amount of the crystalline and amorphous phase is nearly constant, independent of the cold-drawn ratio. The amount of the intermediate phase is also almost constant but the content of gauche and trans segments is strongly influenced by the cold-draw ratio; and a linear variation of the content of trans segments is obtained with the cold-drawn ratio. The mechanical properties of the oriented monofilaments are directly correlated to the properties of the intermediate phase and confirm that the intermediate phase is the linking phase between the crystallites. These results suggest that the elasticity modulus is determined by trans segment in the intermediate phase and that the intermediate phase is related to the tie molecules. It was found that the two peaks in the Raman spectra, respectively, at 1303 and $1295 \mathrm{~cm}^{-1}$, can be correlated to the amount of gauche and transmolecules in the polyethylene monofilaments. A constructive and new insight into the three phase morphology was obtained by combining the DSC and X-ray measurements with the amounts of transand gauche molecules calculated from the Raman spectra analysis.

\section{References}

[1] D. C. Mcfaddin, K. E. Russell, G. Wu, and R. D. Heyding, "Characterization of polyethylenes by $\mathrm{x}$-ray diffraction and ${ }^{13} \mathrm{C}-\mathrm{NMR}$ : temperature studies and the nature of the amorphous halo," Journal of Polymer Science, Part B, vol. 31, no. 2, pp. 175-183, 1993.

[2] A. G. Simanke, R. G. Alamo, G. B. Galland, and R. S. Mauler, "Wide-angle X-ray scattering of random metalloceneethylene copolymers with different types and concentration of comonomer," Macromolecules, vol. 34, no. 20, pp. 6959-6971, 2001.

[3] P. Sajkiewicz, T. Hashimoto, K. Saijo, and A. Gradys, "Intermediate phase in poly(ethylene) as elucidated by the WAXS. Analysis of crystallization kinetics," Polymer, vol. 46, no. 2, pp. 513-521, 2005.

[4] G. R. Strobl and W. Hagedorn, "Raman spectroscopic method for determining the crystallinity of polyethylene," Journal of Polymer Science, Polymer Physics Edition, vol. 16, no. 7, pp. 1181-1193, 1978. 
[5] P. J. In 't Veld, M. Hütter, and G. C. Rutledge, "Temperaturedependent thermal and elastic properties of the interlamellar phase of semicrystalline polyethylene by molecular simulation," Macromolecules, vol. 39, no. 1, pp. 439-447, 2006.

[6] S. Nikolov, "Multi-scale constitutive modeling of the small deformations of semi-crystalline polymers," Journal of the Mechanics and Physics of Solids, vol. 50, no. 11, pp. 2275-2302, 2002.

[7] Z. Špitalský and T. Bleha, "Elastic moduli of highly stretched tie molecules in solid polyethylene," Polymer, vol. 44, no. 5, pp. 1603-1611, 2003.

[8] S. Nikolov and I. Doghri, "A micro/macro constitutive model for the small-deformation behavior of polyethylene," Polymer, vol. 41, no. 5, pp. 1883-1891, 2000.

[9] O. Gueguen, S. Ahzi, A. Makradi, and S. Belouettar, "A new three-phase model to estimate the effective elastic properties of semi-crystalline polymers: application to PET," Mechanics of Materials, vol. 42, no. 1, pp. 1-10, 2010.

[10] X. Guan and R. Pitchumani, "A micromechanical model for the elastic properties of semicrystalline thermoplastic polymers," Polymer Engineering and Science, vol. 44, no. 3, pp. 433-451, 2004.

[11] O. K. Muratoglu, A. S. Argon, R. E. Cohen, and M. Weinberg, "Toughening mechanism of rubber-modified polyamides," Polymer, vol. 36, no. 5, pp. 921-930, 1995.

[12] G. Elsner, J. Kempf, J. W. Bartha, and H. H. Wagner, "Anisotropy of thermal expansion of thin polyimide films," Thin Solid Films, vol. 185, no. 1, pp. 189-197, 1990.

[13] Z. Bartczak, A. S. Argon, R. E. Cohen, and T. Kowalewski, "The morphology and orientation of polyethylene in films of sub-micron thickness crystallized in contact with calcite and rubber substrates," Polymer, vol. 40, no. 9, pp. 2367-2380, 1999.

[14] M. Glotin and L. Mandelkern, "A Raman spectroscopic study of the morphological structure of the polyethylenes," Colloid \& Polymer Science, vol. 260, no. 2, pp. 182-192, 1982.

[15] J. M. Lagaron, N. M. Dixon, W. Reed, J. M. Pastor, and B. J. Kip, "Morphological characterisation of the crystalline structure of cold- drawn HDPE used as a model material for the environmental stress cracking (ESC) phenomenon," Polymer, vol. 40, no. 10, pp. 2569-2586, 1999.

[16] W. Lin, M. Cossar, V. Dang, and J. Teh, "The application of Raman spectroscopy to three-phase characterization of polyethylene crystallinity," Polymer Testing, vol. 26, no. 6, pp. 814-821, 2007.

[17] L. Mandelkern, "The relation between structure and properties of crystalline polymers," Polymer Journal, vol. 17, no. 1, pp. 337-350, 1985.

[18] J. M. Lagarón, S. López-Quintana, J. C. Rodriguez-Cabello, J. C. Merino, and J. M. Pastor, "Comparative study of the crystalline morphology present in isotropic and uniaxially stretched conventional and metallocene polyethylenes," Polymer, vol. 41, no. 8, pp. 2999-3010, 2000.

[19] S. Rabiej, B. Wlodzimierz, and B. Dorota, "The transition phase in polyethylene-WAXS and Raman investigations," Fibres \& Textiles in Eastern Europe, vol. 16, no. 6, pp. 57-62, 2008.

[20] W. G. Hu and K. Schmidt-Rohr, "Characterization of ultradrawn polyethylene fibers by NMR: crystallinity, domain sizes and a highly mobile second amorphous phase," Polymer, vol. 41, no. 8, pp. 2979-2987, 2000. 

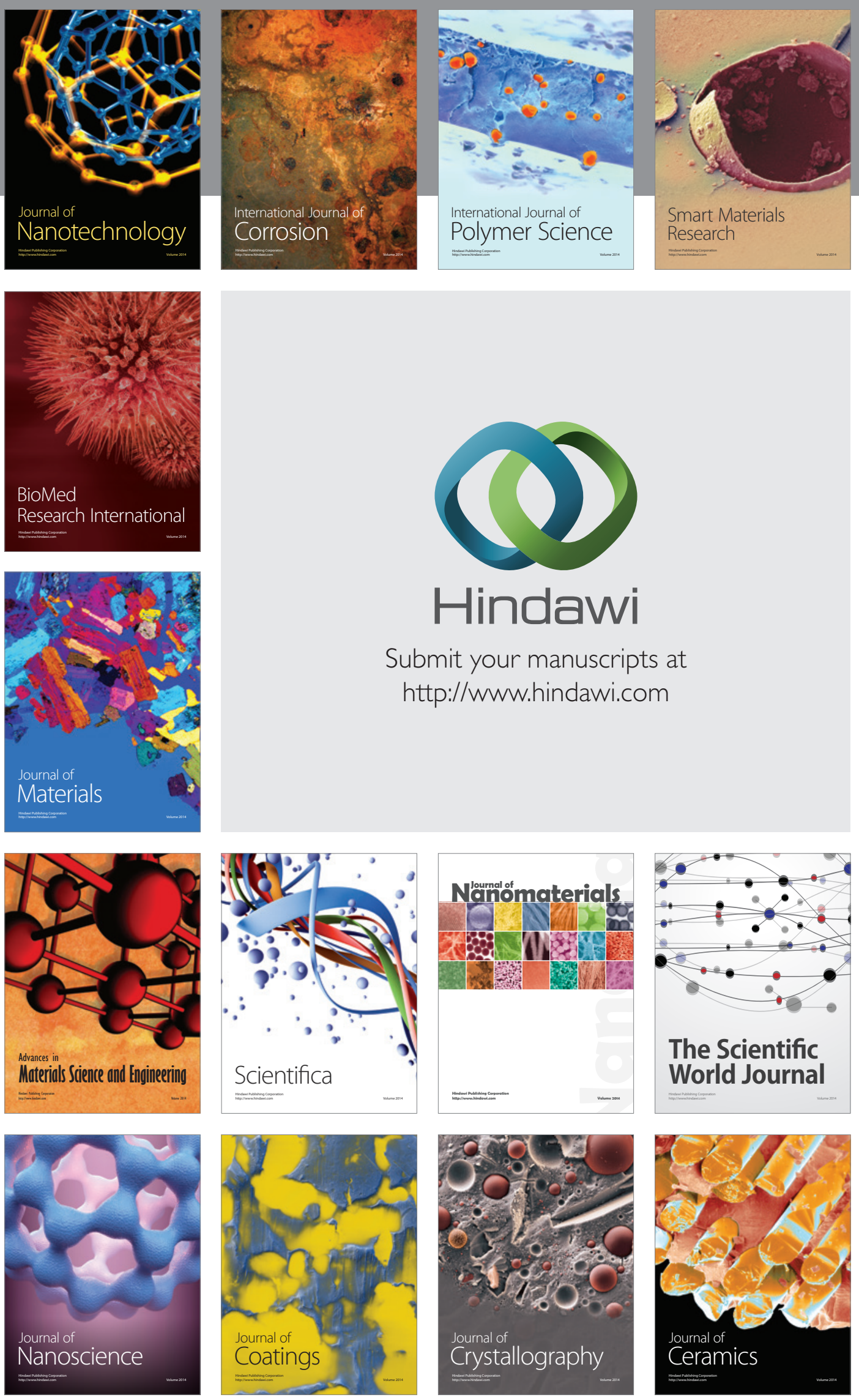

The Scientific World Journal

Submit your manuscripts at

http://www.hindawi.com

\section{World Journal}

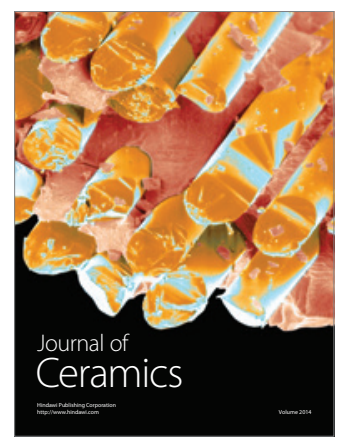

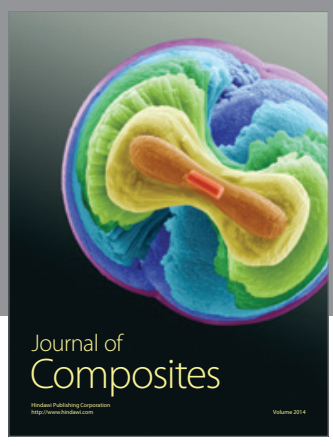
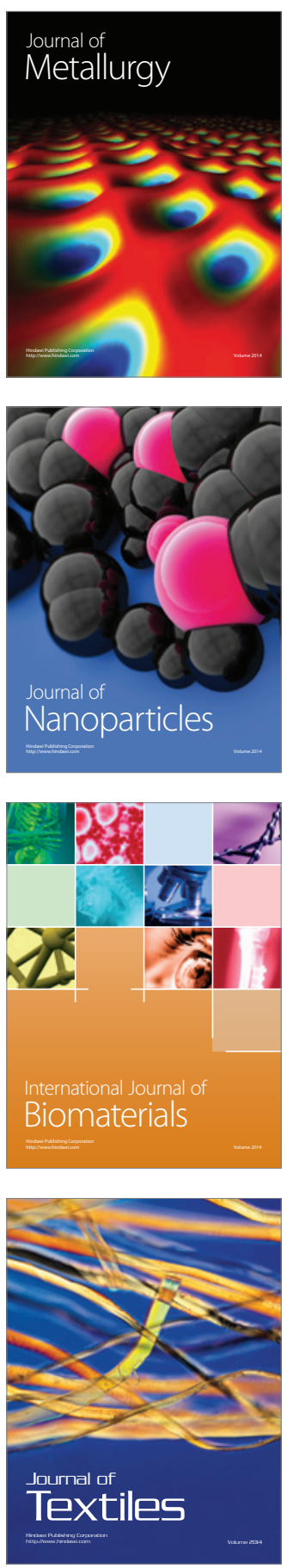